\title{
Counting Materials Researchers
}

\author{
Kathleen C. Taylor \\ MRS President
}

The community of materials researchers is distinguished by the fact that we come to this area of study by many paths. The field derives strength from this diversity of technical backgrounds. Materials researchers have origins in physics, chemistry, metallurgy, mathematics, earth sciences, materials science and engineering, and chemical, electrical, and mechanical engineering.

While many of us have come to call ourselves materials researchers, we have not necessarily moved into a new area. We are still chemists, etc., but we recognize an orientation in our research and application for our scientific findings that is encompassed by the activities of the growing materials community that spans synthesis to properties to performance.

Our diverse origins make counting materials researchers a challenge and perhaps lead to a high underestimation of the size of this community. There are problems associated with underestimation: (1) communication of the needs and opportunities in materials research becomes fragmented, (2) communication of progress is fragmented, and (3) the community can lack the strength and growth potential that comes from a clearly observable identity to young students.

Before we can count materials researchers we need to know what we are. We need a

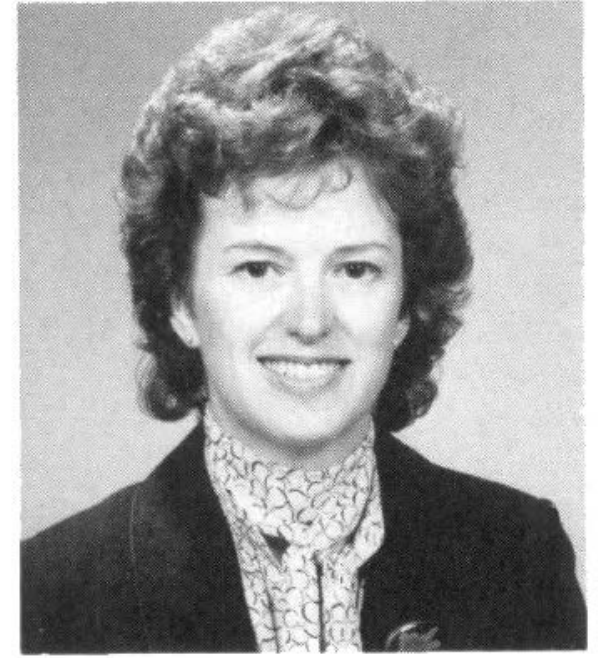

Our diverse origins... perhaps lead to a high underestimation of the size of this community. simple yet comprehensive definition of the area that is widely accepted. Our definition should encompass both the substances we work with and our manipulation of these substances to achieve specific properties. We need to recognize the overlap of materials research with other areas - materials research includes a large part of physics and chemistry.

Overlap between fields and multidisciplinary research projects is currently encouraged by research funding - for example, the NSF-sponsored Materials Research Groups, the Materials Research Laboratories, and the proposed Science and Technology Centers. The growth of job opportunities for materials researchers has stimulated the field. We are all stimulated by significant recent developments in the materials area and the potential for more to come.

The National Research Council survey of the field of Materials Science and Engineering which is currently nearing completion will help clarify who the materials research community is and, moreover, lend needed focus to the field. This focus will make materials researchers recognizable in greater numbers, and allow us to determine more readily the size of the community.

\section{Membership in the Materials Research Society}

The Materials Research Society is dedicated to fostering the exchange of scientific information across the many disciplines involved in materials research. Membership benefits include:

\author{
Subscription to Joumal of Materials Research * \\ Subscription to MRS BULLETIN \\ Discounts on special books published by other leading scientific publishers \\ Discount subscription to Materials Letters \\ Information on upcoming MRS meetings and short courses \\ Copy of the MRS Membership Directory \\ Discount subscriptions on joumals published by the American Institute of Physics \\ *Regular members only. Student members may subscribe for $\$ 15$.
}

To join, contact the Materials Research Society, 9800 McKnight Road, Suite 327, Pittsburgh, PA 15237; telephone (412) 367-3003. 